NANO-OPTICS

\section{Optical antennas tuned to pitch}

\author{
Lukas Novotny
}

\section{Mapping out the resonant modes of optical antennas is part of a largely unexplored terrain - but not any more, as a study that applies a luminescence technique to gold antennas demonstrates.}

Optical antennas are devices designed to efficiently convert optical radiation into localized energy, and vice versa. They enhance the interaction between light and matter, and hence have the potential to boost the efficiency of optoelectronic devices ranging from light-emitting diodes to solar cells. Writing in Physical Review Letters, Ghenuche et al. ${ }^{1} \mathrm{dem}$ onstrate that the resonances of gold antennas can be mapped out spectrally and spatially with a technique known as two-photon excited luminescence (TPL) spectroscopy. The experimental mapping of optical antenna resonances by TPL not only validates theoretical models but also provides an efficient means for visualizing energetically 'hot' regions in arbitrary antenna configurations.

TPL in gold is a weak process that can be enhanced if coupled to surface plasmons ${ }^{2}$, the size and frequency-dependent resonances of a gas of electrons. Regions of high TPL intensity are associated with strong electric fields. Therefore, a spatial map of the intensity describes an antenna's characteristic electricfield distribution. Although TPL has been used before to characterize different forms of optical antennas ${ }^{3-5}$, Ghenuche et al. ${ }^{1}$ provide the first spectrally resolved images; that is, they record spatial maps of the TPL intensity for different excitation wavelengths. The resulting data describe the spatial-spectral evolution of characteristic antenna resonances. In their experiments, the authors focused a pulsed laser beam on optical antennas fabricated by electron-beam lithography and detected the TPL emission of the antennas' response. Spatial maps of the TPL intensity were recorded pixel-by-pixel by raster scanning the antennas through the laser focus.

The optical antennas they studied are gap antennas that consist of gold nanowires separated by a narrow gap of a few nanometres. The resonance wavelength of a single gold nano-rod scales linearly with the antenna length ${ }^{6}$. But the resonance wavelength of a gap antenna also depends sensitively on the gap size $e^{7}$. The gap antennas of Ghenuche et al. exhibited a sharp resonance at an excitation wavelength, $\lambda$, of 730 nanometres, in excellent agreement with the authors' model calculations. This agreement allowed them to identify their measured antenna resonances as higher-order modes, namely as $3 \lambda / 2$ and $5 \lambda / 2$ resonances. Antennas operating at the fundamental resonance $(\lambda / 2)$ can be expected to be more efficient than those fabricated by Ghenuche et al., but they need to be

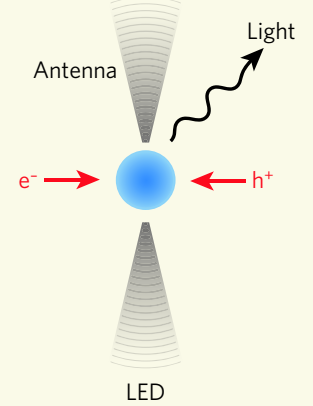

shorter, requiring more stringent fabrication tolerances ${ }^{3}$. Also, an optical antenna interacts with the radiation it generates and it would be interesting to measure the influence of the antenna length on the TPL emission spectrum.

The spatial resolution of spectroscopic TPL imaging is limited by diffraction to about a third of the wavelength of the illuminating laser. But although this resolution can identify the modes of an antenna, higher resolution is needed to fully resolve the details of the energy distribution along the antenna. One possible method for increasing resolution is to use a sharp tip or particle to locally scatter the TPL signal generated by an antenna ${ }^{5}$. The tip is raster scanned over the laser-irradiated antenna in close proximity, and the TPL intensity is measured as a function of the tip's scan coordinates. The resolution of this approach is only limited by the sharpness of the scatterer, and can be as high as 10 nanometres. However, strong interaction between the local scatterer and the antenna must be avoided, so that the antenna's behaviour is not perturbed.

The energy distribution along an optical antenna depends both on the wavelength and on the spatio-temporal properties of the exciting light. For example, the antenna's response depends on whether the excitation laser beam irradiates the entire antenna or only a fraction of it, as is the case in this new study ${ }^{1}$. Similarly, the antenna's response depends on the phase profile of the laser beam and on its polarization state. The use of polarization- and phase-engineered laser pulses will allow a dynamic control of the energy distribution along an optical antenna ${ }^{8}$.

For practical applications, antennas need to be coupled to a source of radiation (transmit- ter) or a sink of radiation (receiver), such as an ion, molecule or quantum dot. Efficient coupling influences the overall behaviour of the antenna, so the antenna and the receiver/transmitter need to be considered as a single system. For example, a gold gap antenna coupled to an impedance-matched source at its gap becomes a half-wave antenna ${ }^{6,9}$ - that is, an antenna that no longer feels the gap. Likewise, the presence of an antenna modifies the properties of the receiver/transmitter. For instance, for an atomic or molecular system, the antenna modifies the transition rates between the different energy states ${ }^{10}$ and, in the case of a strong interaction, it even affects the energy-level structure. Furthermore, the highly localized fields near the antenna open up new interaction mechanisms between light and matter, such as higher-order multipole transitions or momentum-forbidden transitions. These interactions, which are inaccessible in the free space, have the potential to enrich optical spectroscopy and provide new strategies for optical sensing and detection.

The theoretical study of optical antennas is still in its infancy, and we can expect exciting results and developments in the near future. Optical antennas will boost the efficiency of light-matter interactions and enable control of photophysical processes on the nanometre scale. This has many foreseeable applications (Fig. 1), such as high-resolution microscopy and spectroscopy, photovoltaics and photocatalysis, and solid-state lighting.

Lukas Novotny is at the Institute of Optics,

University of Rochester, Rochester, New York 14627, USA.

e-mail: novotny@optics.rochester.edu

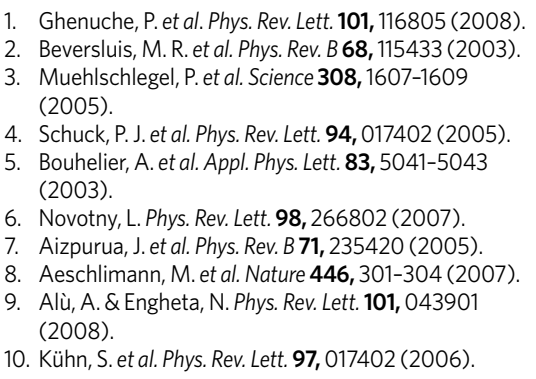

b

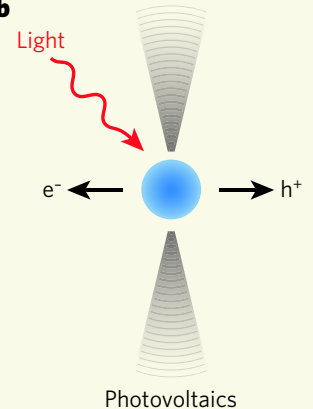

c

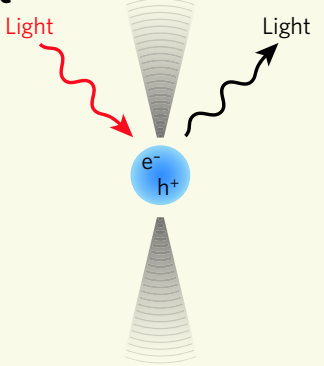

Spectroscopy
Figure 1 Optical antennas and their applications. The gold gap antennas studied by Ghenuche et al. ${ }^{1}$ can find several applications. a, In a light-emitting diode (LED), charge carriers - electrons $\left(\mathrm{e}^{-}\right)$and electron holes $\left(h^{+}\right)$- are recombined in a medium (blue) to produce light. $b$, In photovoltaics, incident light causes separation of the charge carriers. c, In spectroscopy, incident light polarizes the medium of interest, and this polarization gives rise to outgoing radiation. In all three cases, the optical antenna (grey) enhances the efficiency of the input-output conversion process. 